\title{
Molecular Basis of the Activation and Dissociation of Dimeric PYL2 Receptor in Abscisic Acid Signaling ${ }^{\dagger}$
}

\author{
Chuankai Zhao, ${ }^{a}$ and Diwakar Shukla ${ }^{*, a, b, c, d, e, f}$
}

Phytohormone abscisic acid (ABA) is essential for plant responses to biotic and abiotic stresses. Dimeric receptors are a class of $A B A$ receptors that are important for various $A B A$ responses. While extensive experimental and computational studies have investigated these receptors, it remains not fully understood how ABA leads to their activation and dissociation for interaction with downstream phosphatase. It also remains unknown how networks of water molecules present in the binding site affect ABA perception despite its critical role in protein-ligand binding. Here, we study the activation and the homodimeric association processes of PYL2 receptor as well as its heterodimeric association with the phosphatase HAB1 using molecular dynamics simulations. Free energy landscapes from $\sim 223 \mu$ s simulations show that dimerization substantially constrains PYL2 conformational plasticity and stabilizes inactive state, resulting in lower ABA affinity. Using hydration site analysis to characterize receptor solvation thermodynamics, we show that the displacement and reorganization of water molecules upon ABA binding contribute to binding affinity via gain of entropy and enthalpy, respectively. The penalty for expelling water molecules into the bulk causes the free energy barrier to binding $(\sim 4-5 \mathrm{kcal} / \mathrm{mol})$. Finally, we establish the thermodynamic model for competitive binding between homodimeric PYL2 association and heterodimeric PYL2-HAB1 association in the absence and presence of ABA. Our results suggest that the binding of ABA destabilizes PYL2 complex and further stabilizes PYL2-HAB1 association, thereby promoting PYL2 dissociation. Overall, this study explains several key aspects on activation of dimeric ABA receptors, which provide new avenues for selective regulation of these receptors.

\section{Introduction}

Abscisic acid (ABA) is a vital plant hormone that responds to a variety of environmental stresses, including drought which significantly impacts crop yield worldwide. ${ }^{112}$ When plants are under water deficiency, in planta biosynthesis of ABA is promoted ${ }^{3}$ and elevated levels of $\mathrm{ABA}$ eventually leads to stomata closure, thereby reducing transpiration water loss. ${ }^{4} \mathrm{ABA}$ responses are controlled by a negative regulatory signaling network that involves soluble PYR/PYL/RCAR (pyrabactin resistance1/PYR1like/regulatory component of $\mathrm{ABA}$ receptor) $\mathrm{ABA}$ receptors, PP2Cs (clade A serine/threonine protein phosphatases 2C), and SnRK2s (subfamily 3 SNF1-related kinase 2). $\frac{[5}{5}$ In the absence of ABA, PP2Cs dephosphorylate and inactivate SnRK2s. ${ }^{6 / 7}$ When

\footnotetext{
${ }^{a}$ Department of Chemical and Biomolecular Engineering, University of Illinois at Urbana-Champaign, Urbana, IL 61801, USA. E-Mail: diwakar@illinois.edu, Tel: +1 217-300-0021.

${ }^{b}$ Department of Plant Biology, University of Illinois at Urbana-Champaign, Urbana, IL 61801, USA.

${ }^{c}$ Center for Biophysics and Quantitative Biology, University of Illinois at UrbanaChampaign, Urbana, IL 61801, USA.

${ }^{d}$ National Center for Supercomputing Applications, Urbana, IL 61801, USA.

${ }^{e} \mathrm{NIH}$ Center for Macromolecular Modeling and Bioinformatics, University of Illinois at Urbana-Champaign, Urbana, IL 61801, USA.

${ }^{f}$ Beckman Institute for Advanced Science and Technology, University of Illinois at Urbana-Champaign, Urbana, IL 61801, USA.

$\dagger$ Electronic Supplementary Information (ESI) available online: methods, supplementary tables and figures.
}

ABA binds to PYR/PYL/RCAR receptors, these receptors inactivate PP2Cs via stable binding and prevent SnRK2s from dephosphorylation by PP2Cs. ${ }^{8}$ SnRK2s then activate through autophosphorylation, which triggers the downstream signaling cascade.9-11] PYR/PYL/RCAR receptors have been demonstrated as promising chemical and genetic targets for improving plant water use efficiency. ${ }^{[12 \mid 15}$ Notably, a series of ABA agonists covering various chemotypes ${ }^{[-16-20}$ and a variety of engineered $A B A$ receptors ${ }^{2122}$ have been discovered in recent years, in order to achieve selective control of PYR/PYL/RCAR receptor activity in multiple plant species.

Since 2009, the gate-latch-lock mechanism for explaining the mode of action of ABA has been established from crystallographic structural studies on ABA receptors. ${ }^{23-26}$ In Arabidopsis thaliana, PYR/PYL/RCAR receptors can be classified according to their oligomeric states in solution: PYR1, PYL1 and PYL2 form a stable homodimer, while PYL4-12 are monomeric and PYL3 is in equilibrium between monomeric and dimeric states. $.2425 / 27,28$ All receptors share high sequence and structural similarities, and $\mathrm{ABA}$ binds to a structurally conserved hydrophobic binding pocket which is filled by water molecules. ${ }^{24}$ The binding of ABA is stabilized by both direct and water-mediated hydrogen bonding interactions with several polar residues at bottom of the binding site as well as hydrophobic interaction with other nonpolar residues. ${ }^{24}$ Upon ABA binding, two flexible loops at top of the binding cavity, named as the gate loop and the latch loop, will 
undergo large conformational changes to close and activate ABA receptors. 23 The closed gate loop creates interaction interface to facilitate the binding between ABA receptors and PP2Cs. The monomeric receptors show ABA-independent constitutive activity, 2729 whereas the dimeric receptors are ABA-dependent and have nearly two orders of magnitude lower ABA affinity. ${ }^{[30}$ When PP2Cs bind to ABA-bound receptors, PP2Cs form water-mediated interaction with the carbonyl group of ABA. ${ }^{23}$ Therefore, PP2Cs are often considered as ABA co-receptors. 31

While a large number of experimental studies have probed the ABA signaling mechanism, the atomic-scale dynamics of ABA perception and subsequent conformational changes in $\mathrm{ABA}$ receptors remained elusive. Specifically, it was unclear how the binding of ABA happens and leads to the activation of PYR/PYL/RCAR receptors, and how these processes vary among different receptors. In our recent work ${ }^{32}$, we have employed molecular dynamics (MD) simulations to investigate ABA-mediated activation processes of monomeric PYL5 and PYL10 receptors belonging to two separate clades of PYR/PYL/RCAR receptors in Arabidopsis thaliana. Strikingly, the gate loop remains flexible between open and closed conformations after ABA binding, suggesting that $\mathrm{ABA}$ binding is necessary but insufficient for full activation of monomeric receptors. PP2C binding stabilizes the closed gate loop conformation, which explains enhanced ABA affinity in the presence of PP2C.33 In contrast to PYL5, the gate loop in PYL10 can close without ABA binding, which unravels the PYL10 ABA-independent activation mechanism.2729

Several key questions remain unanswered regarding the activation mechanism of dimeric ABA receptors. Previous studies have shown that dimeric receptors are critical for $A B A$ responses, despite their lower ABA sensitivity compared to monomeric receptors. For example, activation of dimeric receptors by selective ABA agonists has led to guard cell closure in both soybean and Arabidopsis thaliana.16/18 While dimeric receptors such as PYL2 are inactive in the absence of $\mathrm{ABA}$, it was shown that monomeric variant of PYL2-I88K obtained weak constitutive activity on PP2C inhibition. .27 Furthermore, ABA binding affinity to PYL2-I88K is 7-fold higher compared to wild-type PYL2 as measured by isothermal titration calorimetry. 27 What is the molecular origin of intrinsic $A B A$ affinity differences between monomeric and dimeric receptors? How does ABA bind to dimeric receptors and mediate their conformational changes? Since ABA-bound receptors form a 1:1 heterodimer with PP2Cs, dimeric receptors need to dissociate after ABA binding to inhibit PP2Cs. However, PYR1 and PYL1-2 remain in a dimer conformation in the presence of ABA suggested by a range of experimental characterizations, including size exclusion chromatography, small angle x-ray scattering and analytical ultracentrifugation experiments. 2526262830 It was noted that the gate loop closure caused by ABA binding may weaken dimeric receptors by comparing the structures of ABA-free and ABA-bound PYL2 crystal structures. ${ }^{26}$ It is unclear how $A B A$ affects the assembly of dimeric receptors and the heterodimeric association between these receptors and PP2Cs. Thus, the molecular mechanism of activation and dissociation of dimeric $\mathrm{ABA}$ receptors requires further investigation.

Another aspect in understanding $\mathrm{ABA}$ receptor activity that remains largely unknown is the role of solvation structure and thermodynamics of the binding site on $\mathrm{ABA}$ perception. It has been well recognized that water molecules play an important role in protein-ligand binding. ${ }^{34 \sqrt[36]{36}}$ The thermodynamics and phase behavior of water molecules in the binding cavity of proteins can have substantial differences from that in the bulk. ${ }^{[34}$ Upon ligand binding, water molecules in the binding site are displaced into the bulk and the accompanying changes in enthalpy and entropy of these displaced water molecules usually significantly contribute to overall free energy of ligand binding. In addition, water molecules that remain in the bound complex restructure which leads to further thermodynamic consequences. It has been demonstrated in many applications that manipulation of water displacement and reorganization can dramatically enhance protein-ligand binding affinity. $\frac{[37,40}{40}$ Therefore, the determination of whether water molecules in ABA receptors maintain favorable interaction with its neighboring water and protein is critical in understanding the drive force of ligand binding and potentially optimizing ligands for enhanced affinities.

In this work, we seek to understand the molecular mechanism of activation and dissociation of dimeric ABA receptors as well as the role of water on $\mathrm{ABA}$ perception using various computational approaches. We focus on PYL2 receptor in this study. First, we have utilized extensive all-atom MD simulations ( $\sim 223$ $\mu$ s aggregate) to elucidate the molecular basis of ABA-mediated PYL2 activation in both its monomeric and dimeric forms. Using Markov state models (MSMs) to analyze massive simulations, 41,45 we elucidated the entire pathways of ABA binding and activation of both PYL2 receptors, along with quantitative thermodynamic and kinetic characterizations. Our results have shown that the homodimer conformation substantially stabilizes the open gate loop (inactive-like) conformation, thereby resulting in lower ABA affinity. The free energy landscapes suggest a large barrier $(\sim 4-5 \mathrm{kcal} / \mathrm{mol})$ for ABA binding, consistent with our observations in both PYL5 and PYL10. $\frac{32}{32}$ Using inhomogeneous solvation theory-based hydration site analysis,, 3437146 we have elucidated the solvation structure and thermodynamic properties of the binding site in both apo and holo PYL2. Our results show that the displacement of water molecules during ABA binding contribute to free energy of binding mostly via gain of entropy of released water molecules. The binding of ABA stabilizes the restructured water network in the complex, which leads to further enthalpy gain in free energy of binding. Interestingly, ABA binds to PYL2 via a pathway with the minimal penalty for exclusion of water molecules from the cavity, and the exclusion of water result in the accumulative barrier to ABA binding. To understand the role of ABA in homodimeric PYL2 association and heterodimeric PYL2-PP2C association, we have utilized replica exchange umbrella sampling (REUS) simulations ${ }^{47}$ and multistate Bennett acceptance ratio (MBAR) method ${ }^{48}$ to accurately estimate the standard association free energies for both cases.49 51 Based on our free energy calculation results, we establish a ther- 
modynamic model to explain the competitive interactions between PYL2 self-association and its association with the downstream phosphatase HAB1. Our results suggest that the binding of ABA causes $\sim 5 \mathrm{kcal} / \mathrm{mol}$ difference in PYL2 association free energy, thereby destabilizing PYL2 complex. Furthermore, the binding of ABA significantly enhances the association between PYL2 and HAB1 $(\sim 8 \mathrm{kcal} / \mathrm{mol})$, thereby further promoting PYL2 dissociation. Overall, this study provides key molecular insights into the activation of an important class of ABA receptors in drought resistance signaling, which can be exploited for future genetic and agrochemical control of ABA-regulated stress responses.

\section{Results and discussion}

Simulations reveal the conformational landscapes of the monomeric and the dimeric PYL2.

We have performed $\sim 107 \mu$ s and $\sim 116 \mu$ s (aggregate) adaptive MD simulations ${ }^{52 \sqrt[54]{54}}$ to study $\mathrm{ABA}$ binding and subsequent conformational changes in both the monomeric and the dimeric PYL2 (Tables S1 and S2). While PYL2 only exists as a dimer in solution, we study the dynamics of both the monomeric and the dimeric states in order to investigate how the dimerization affects PYL2 activation. All simulations are initialized from the inactive PYL2 crystal structure (PDB: $3 \mathrm{KDH}^{26}$ ). In both cases, a single ABA molecule is present in the MD simulations. For the dimeric PYL2, our simulations have captured the binding of ABA to one of the protomers in PYL2. All the conformations collected from MD simulations are clustered into 300 and 200 states for the monomeric and the dimeric PYL2, respectively. A set of distance features are used for clustering analysis, which include the metrics that describe the conformations of both the gate loop and the latch loop, ABA position and PYL2-ABA interactions (Table S3). MSMs are then built to estimate the equilibrium probabilities of all states and their inter-state transition probabilities. The thermodynamics and kinetics of ABA binding and receptor activation processes can be inferred from these MSMs. ${ }^{42 / 43}$ Details of the simulations and analysis are summarized in Supplementary Methods.

We report the conformational free energy landscapes for both the monomeric and the dimeric PYL2 receptors (Fig. 1 $1 \mathrm{~A}, \mathrm{~B}$ ) as well as the associated error bars on the landscapes (less than 0.4 $\mathrm{kcal} / \mathrm{mol}$, Fig. S1A, B). The $x$ and $y$ metrics of the landscapes clearly distinguish whether ABA is bound to PYL2 and whether the gate loop is in open or closed states, respectively. The free energy landscapes of both receptors share similar minima, which correspond to the binding intermediate states and the ABA-bound states. Before ABA binds, there is only a single flattened minima on both the landscapes, with the gate loop RMSD centering around $\sim 4-5 \AA$ indicating an open gate loop conformation. The free energies of these binding intermediate states are $0-2 \mathrm{kcal} / \mathrm{mol}$, suggesting that they are relatively stable conformations. However, the gate loop RMSD from the active structure fluctuates between 1-11 $\AA$ for the monomeric PYL2, whereas the gate loop RMSD for the dimeric PYL2 only fluctuates between 2-8 $\AA$. These results show that breaking the PYL2 complex results in a higher degree of conformational flexibility of the gate loop. Since the gate loop in monomeric PYL2 may adopt a closed conformation before ABA binds, the monomeric variant of PYL2 receptor would exhibit weak constitutive activity in solution. Our results are in agreement with the finding that the monomeric PYL2-I88K exhibited an increased constitutive activity on PP2C inhibition compared to the dimeric PYL2. 27

After ABA binds, we observe two minima that correspond to the open and closed gate loop conformations. The landscapes clearly suggest that the binding of ABA promotes the gate loop closure for both the monomeric and the dimeric receptors. For the monomeric PYL2, the free energies for the open gate loop and the closed gate loop states are nearly comparable $(<2 \mathrm{kcal} / \mathrm{mol})$, suggesting that the gate loop is in equilibrium between the open and the closed conformations after ABA binding. This is consistent with our previous finding on the monomeric PYL5 and PYL10 receptors that $\mathrm{ABA}$ binding is necessary but insufficient for full receptor activation. ${ }^{32}$ In contrast to the monomeric PYL2 receptor, the free energy of the open gate loop state for the dimeric PYL2 $(<2 \mathrm{kcal} / \mathrm{mol})$ is lower as compared to that of the closed gate loop state $(\sim 3 \mathrm{kcal} / \mathrm{mol})$. In other words, the open gate loop conformation is stabilized due to the formation of dimer structure. Our results are further supported by the hydrogen/deuterium exchange (HDX) mass spectrometry data that high levels of HDX for the gate loop in PYL2 are observed both in the presence and in the absence of ABA. ${ }^{[55}$ Interestingly, we have observed large free energy barriers for $\mathrm{ABA}$ binding to both the monomeric and the dimeric PYL2 receptors, which are $\sim 5$ and $\sim 4 \mathrm{kcal} / \mathrm{mol}$ respectively. The binding free energy barriers in PYL2 are comparable

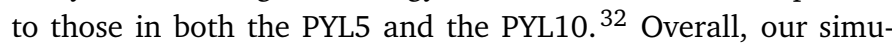
lations show that $\mathrm{ABA}$ is required for PYL2 activation, and the dimeric PYL2 constrains the gate loop conformational plasticity and destabilizes the closed gate loop (active-like) conformation.

\section{Transition path theory characterizes the pathway of ABA binding and PYL2 activation.}

To obtain structural insights into PYL2 activation processes, we use transition path theory (TPT) to determine the pathways associated with $\mathrm{ABA}$ binding and the conformational changes in both the monomeric and the dimeric receptors. .5657 The transition states in the top pathways are mapped onto the landscape and labelled as states 1-5 (Fig. 17A, B). The snapshots of these states are shown in Fig. 1C and Fig. S1C, with the gate loop and the key interactions between PYL2 and ABA highlighted.

Our results suggest that $\mathrm{ABA}$ binds to both monomeric and dimeric PYL2 receptors through a series of similar transition states, despite minor differences due to dimerization. The initial interaction between PYL2 and ABA is mediated by a direct contact between R120 and the carboxylate group of ABA, while the gate loop is open (state 1, Fig. 1 1 ). Next, ABA enters into top of the binding pocket and interacts with both protomers, resulting in the stable intermediate state 2 (Fig. 13). Specifically, the carboxylate group of ABA forms hydrogen bonding interactions 
A

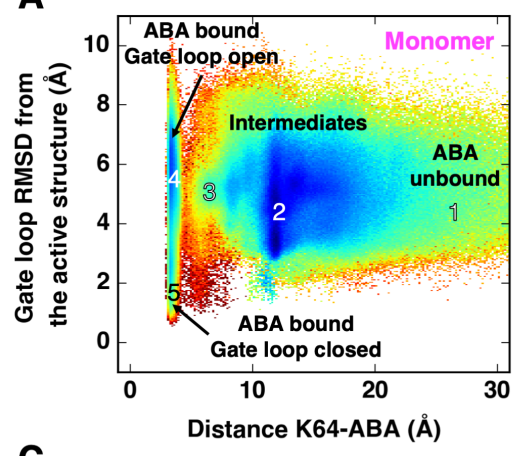

B

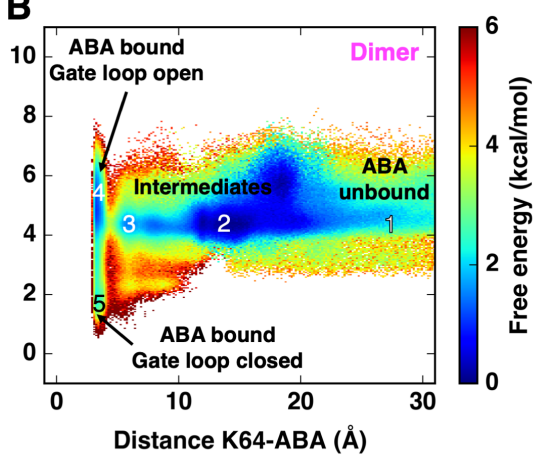

3 L91,

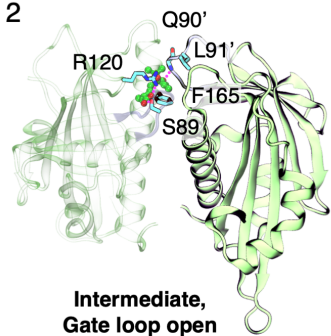

Gate loop open

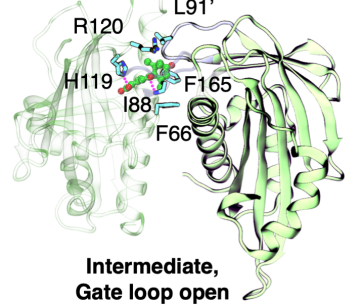

Gate loop open

5

4

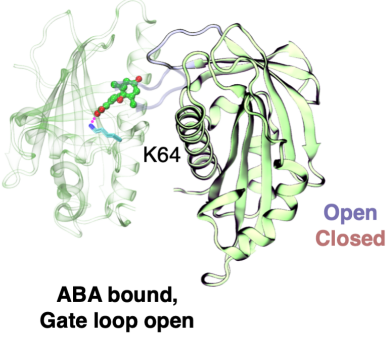

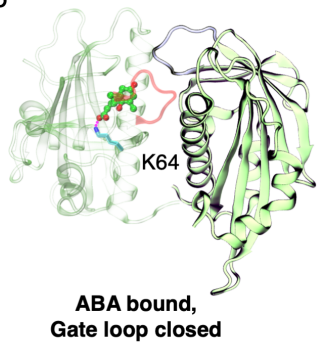

Fig. $1 \mathrm{ABA}$ binding and subsequent PYL2 activation processes. Free energy landscapes of $A B A$ binding to $(A)$ the monomeric and $(B)$ the dimeric PYL2 receptors. The landscapes are generated by projecting all the conformations onto two metrics, the distance between $A B A$ and the binding site (K64 in PYL2) as well as the gate loop RMSD from the PYL2 active crystal structure (PDB: 3KDI26), weighted by MSM probabilities. K64-ABA distance is measured as distance between the carbon atom in $-\mathrm{COO}^{-}$group of $\mathrm{ABA}$ and the $\mathrm{NZ}$ atom in $-\mathrm{NH}_{3}^{+}$group of $\mathrm{K} 64$. The $C_{\alpha}$ atoms of $\mathrm{V} 87-\mathrm{S} 92$ in PYL2 are used for calculating RMSD of the gate loop. (C) Snapshots of the states 1-5 corresponding to the minima on the dimeric PYL2 free energy landscape. Key residues that interact with ABA are shown, and the gate loops are highlighted in iceblue (open) or red (closed).

with the side chains of R120 and S89 in one protomer, while the carbonyl group of ABA interacts with the backbone of Q90' in the other subunit (state 2, Fig. 17). Furthermore, the ring of ABA is sandwiched by F165 and L91', which stabilizes ABA via $\pi-\pi$ and hydrophobic interactions. We note that the interactions of ABA with Q90' and L91' can only occur in the dimeric PYL2 but not in the monomeric PYL2. Then, the carboxylate group of ABA moves towards the binding site to reach the intermediate state 3. At the state 3 (Fig. 1 1 ), the carboxylate group and the hydroxyl group of ABA contact with the side chain of H119 and the backbone of I88, in addition to the hydrophobic interactions between ABA and several residues (F66, R120, F165, L91'). Both the gate loops remain open even after $\mathrm{ABA}$ reaches the binding site and forms a hydrogen bond with K64 (state 4, Fig. 1C). Finally, the gate loop in the ABA-bound protomer closes, while the gate loop in the ABA-free protomer remains open (state 5, Fig. 1 1 C). Previous study suggested that mutations in the binding pocket (K64A, F66A, F165A), the gate (S89A, L91A) and the latch (H119, R120) loops resulted in attenuated in vitro PYL2 activity. $\underline{23}$ The activation pathway identified from our simulations highlights the critical roles of these residues in ABA binding.

Despite the similar activation pathways of both receptors, we note that there are key differences in relative stability of several transition states which depend on PYL2 oligomeric state (Fig. 17A, B). Notably, the intermediate state 3 is more stable in the dimeric PYL2 while the ABA-bound, closed gate loop state 5 is much less stable, compared to the monomeric PYL2. The free energy of state 3 in the dimeric PYL2 is $\sim 2 \mathrm{kcal} / \mathrm{mol}$, which is more stable (at least $1 \mathrm{kcal} / \mathrm{mol}$ in free energy difference) compared to that in the monomeric PYL2. The gain in stability is attributed to the dimer structure, where both protomers of PYL2 interact with ABA. In contrast, the state 3 in the monomeric PYL2 is less stable due to the weakened hydrophobic interaction between PYL2 and ABA (Fig. S1C). As discussed above, the state 5 in the dimeric PYL2 is less stable, which suggests that the dimerization 


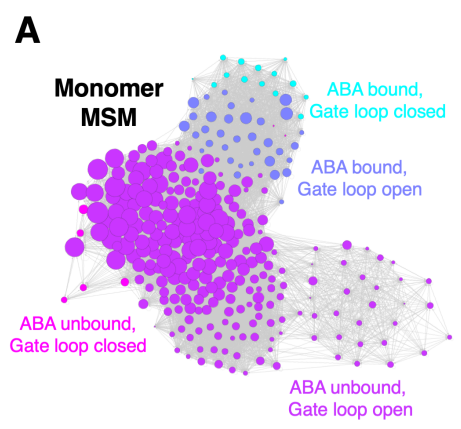

B

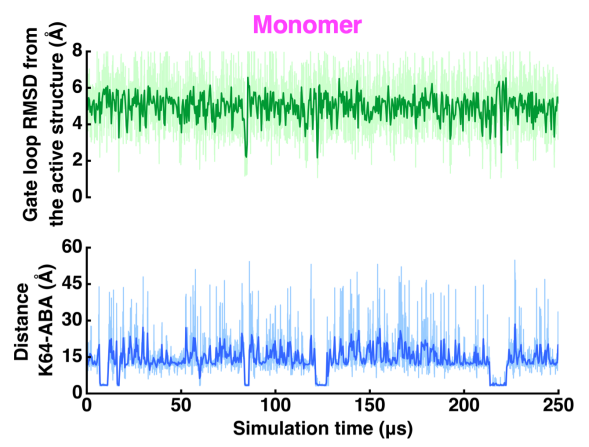

C

D
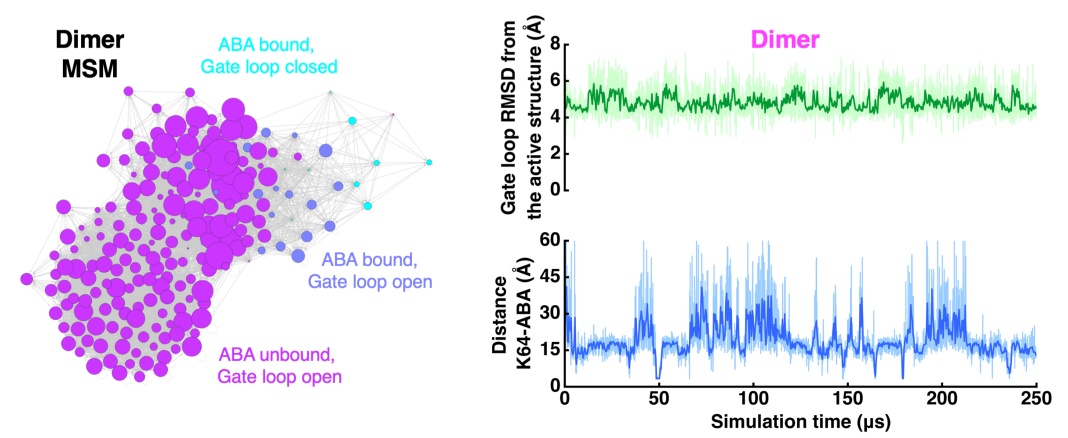

Fig. 2 Kinetics of ABA binding to the monomeric and the dimeric PYL2 receptors. The conformational networks describe the ABA-mediated activation processes for $(A)$ the monomeric and $(C)$ the dimeric receptors. The dots represent the individual conformational states in the MSMs, scaled by the square roots of their MSM probabilities. The grey edges represent the transitions between these states. Long timescale dynamics of (B) the monomeric and (D) the dimeric PYL2 revealed by the conformational networks, showing the ABA binding and unbinding events (blue), the gate loop fluctuations (green) and the timescales at which they occur.

makes PYL2 less likely to adopt the closed gate loop, active-like conformation.

The binding poses of ABA and the closed gate loop conformations predicted from our MD simulations closely match with the active PYL2 crystal structure (PDB: 3KB3 ${ }^{23}$ ) (Fig. S1D). Furthermore, we have captured the similar water-mediated interaction networks as in the crystal structure (Fig. S1E, F). Overall, our simulations elucidate the complete pathways of ABA binding and conformational changes in PYL2, and suggest that the formation of dimeric complex stabilizes the binding intermediate state.

\section{Dimeric PYL2 stabilizes open gate loop conformation and lowers ABA binding affinity.}

The conformational transition networks in both monomeric and dimeric PYL2 estimated from the MSMs are shown in Fig. 24A, C. All circles correspond to the MSM states, scaled by their equilibrium probabilities. We classify these MSM states into 4 different categories based on whether ABA is bound or unbound (4 $\AA$ as the cutoff PYL2-ABA distance) and whether the gate loop is open or closed ( $3 \AA$ as the cutoff gate loop RMSD). It is clearly shown that the gate loop in the dimeric PYL2 can only close after ABA is bound, highlightling a ABA-dependent activation process. However, for the monomeric PYL2 receptor, the population of closed gate loop states is small when ABA is not bound 27. In both cases, the binding of $\mathrm{ABA}$ enhances the equilibrium populations of closed gate loop states. Therefore, the binding of $\mathrm{ABA}$ is necessary for initializing the dimeric PYL2 activation.

Next, we seek to characterize the long-timescale dynamics of monomeric and dimeric PYL2. From kinetic Monte Carlo simulations ${ }^{58}$ on the conformational networks, we generate $250 \mu$ s long trajectories staring from an arbitrary ABA-unbound, open gate loop state. For both the receptors, we have captured multiple ABA binding and unbinding events, and the associated conformational changes in the gate loop (Fig. 2B, D). It is apparent that the fluctuations in the gate loop conformation are significantly reduced in the dimeric PYL2 compared to the monomeric PYL2. When ABA is unbound, the gate loop RMSD primarily fluctuates between 3-8 $\AA$ for monomeric PYL2, and 4-7 $\AA$ for dimeric PYL2. When ABA is bound, for monomeric PYL2, the gate loop RMSD fluctuates between 1-8 Å suggesting that the gate loop closure is promoted by ABA binding but the loop remains flexible between open and closed conformations. However, for dimeric PYL2, we rarely observe that the gate loop RMSD reaches below $2 \AA$ even after ABA is bound, suggesting that the stability of closed gate loop conformation decreases due to dimerization. We also note that the residence time of $A B A$ in the bound state is substantially reduced by an order of magnitude for dimeric PYL2. In other words, ABAbound pose is relatively less stable in dimeric PYL2 compared to monomeric PYL2. Thus, we conclude that the dimeric PYL2 stabilizes the open gate loop conformation and thereby lowers $\mathrm{ABA}$ 
binding affinity.

\section{Displacement and reorganization of water molecules upon ABA binding contribute to free energy of ABA binding.}

To investigate how water molecules in PYL2 affect ABA binding, we seek to characterize solvation structural and thermodynamic properties at the binding site. Explicit solvent MD simulations on PYL2 receptor without (denoted by apo) and with (denoted by holo) ABA are performed for 100 ns with restraints applied on protein backbone, followed by hydration site analysis (HSA). 34/37/46 We use 100,000 frames of MD trajectory data to perform HSA calculations. HSA calculates structural and thermodynamic properties of water molecules in the binding cavity that are represented by a set of high-occupancy $1 \AA$ spheres, known as hydration sites. $\frac{37}{3}$ These sites are identified by clustering the solvent density distribution sampled from explicit solvent MD simulations. Inhomogenous solvation theory 46 is applied to estimate the thermodynamic properties for water molecules in these sites. The major thermodynamic properties calculated by HSA include both system interaction energy $\left(E_{t o t}\right)$ and excess entropy relative to bulk water $\left(-T S^{e}\right) . \quad E_{t o t}$ is further broken down into solute-water $\left(E_{s w}\right)$ and water-water $\left(E_{w w}\right)$ interaction energies. $E_{w w}$ includes contribution from different solvation shells. The average water-water interaction energy per neighbor $\left(E_{w w}^{n b r}\right)$, the contribution from the first solvation shell divided by the number of neighbors in the first shell $\left(N_{n b r}\right)$, measures enhancement or frustration in the local water interactions for each hydration site. Several structural quantities are reported for each hydration site, including the numbers of solute-water $\left(N_{s w}^{H B}\right)$ and water-water $\left(N_{w w}^{H B}\right)$ hydrogen bonds, the fraction of hydrogen-bonded neighbors $\left(f_{w w}^{H B}\right)$ and the fractional enclosure $\left(f_{\text {enc }}\right)$ that indicates degree of blocked contact with other water. Tables S4 and S5 include the structural and thermodynamic data calculated for apo and holo hydration sites. The convergence of HSA calculations is shown in Fig. S2A,B.

In the apo PYL2 receptor, we have identified 30 hydration sites, and their thermodynamic and structural quantities are reported and compared to bulk water properties ${ }^{59}$ (Table S4). The hydration sites are indexed from 0-29, ranked by their occupancies $\left(f_{o}\right)$. Upon ABA binding, water molecules in the hydration sites are displaced into the bulk, which contribute to free energy of binding due to the differences in enthalpy and entropy of water molecules between the bulk and the binding site. Compared to $E_{\text {tot }}$ in the bulk, 17 hydration sites have more favorable interactions and 13 hydration sites have unfavorable interactions (Fig. 3A). Displacement of water molecules in the unfavorable hydration sites result in enthalpic gain for enhanced affinity and less penalty for binding. In contrast, displacement of water molecules in the favorable sites can lead to enthalpic cost for decreased affinity and large penalty for binding. All the sites are entropically less stable compared to the bulk (Fig. 3A), and exclusion of water molecules result in entropic gain for enhanced affinity. Generally, the hydration sites with stronger solute-water interactions have weaker water-water interactions (Fig. 3B). Compared to $E_{w w}^{n b r}$ of bulk water, 20 sites have enhanced water structure with higher $E_{s w}$ and 10 sites have frustrated water structure with lower $E_{s w}$. Based on both $E_{t o t}$ and $E_{w w}^{n b r}$, the hydration sites can be classified into 4 types: 8 enhanced favorable sites (En.F), 9 frustrated favorable sites (Fr.F), 12 enhanced unfavorable sites (En.U), and 1 frustrated unfavorable sites (Fr.U). These results provide a detailed view of the structural and thermodynamic properties of hydration in the receptor binding site.

The enthalpic and entropic contribution of displacing water molecules to the bulk upon ABA binding to overall free energy of binding are quantified (Fig. 3D, E). The hydration sites that are within $2 \AA$ of any atom of ABA in the binding site are considered to be displaced by ABA. Similarly, the hydration sites that are within $2 \AA$ of any atom of the gate loop and the latch loop in the active state are considered to be displaced by the loops. By comparing with the hydration sites captured in holo PYL2 receptors (Fig. 3F, G), the hydration sites that conserved in both apo and holo PYL2 are also identified. Using these criteria, we have identified 10 conserved sites, 9 sites excluded by ABA and 5 hydration sites that are displaced by the loops, and 6 other sites that remain elusive. We first focus on the 9 hydration sites that are displaced by $A B A$ and their structural and thermodynamic properties are summarized in Table 1. Generally, these displaced water molecules are less energertically favorable relative to the conserved hydration sites (Fig. 3D). Except for the site 21, all the hydration sites exhibit enhanced local water structure relative to the bulk, and $E_{w w}$ contributes mostly to $E_{t o t}$. Among the 9 hydration sites, 6 sites (indices 13, 15, 18, 19, 21, 25) have nearly comparable $E_{t o t}$ to the bulk, which will have little enthalpic contribution to binding affinity. Two hydration sites (indices 14 and 24) are slightly more energetically stable (-0.45 and $-0.41 \mathrm{kcal} / \mathrm{mol}$ differences in $E_{t o t}$ ) compared to bulk, which can cause some penalty and enthalpic cost for ABA binding. The hydration site 20 is energetically unfavorable $(+1.06 \mathrm{kcal} / \mathrm{mol}$ difference in $E_{t o t}$ ), thereby displacing water molecule in site 20 can result in enthalpy gain for enhanced affinity. The net enthalpic contribution of excluding all water molecules to the bulk is $-0.37 \mathrm{kcal} / \mathrm{mol}$, which is nearly negligible. On the other hand, the exclusion of water molecules in these sites can have large entropic contribution to free energy of binding (-37.11 $\mathrm{kcal} / \mathrm{mol})$. Overall, these results suggest that the excluded water molecules by the binding of ABA lead to significant entropic contribution to binding free energy.

The thermodynamic contribution of restructuring water molecules in the binding site after ABA binding to overall free energy of binding can also be quantified by comparing the hydration sites from apo and holo simulations. From holo HSA calculations, we have identified 11 hydration sites (Table S5) that 10 of them are conserved in apo PYL2 (Fig. 3F, G). The additional holo hydration site 4 is not captured in apo due to conformational differences. Among the 10 conserved apo hydration sites, 7 sites (indices $0,1,3-6,10$ ) are energetically favorable but entropically significantly unfavorable compared to the bulk water, which are 

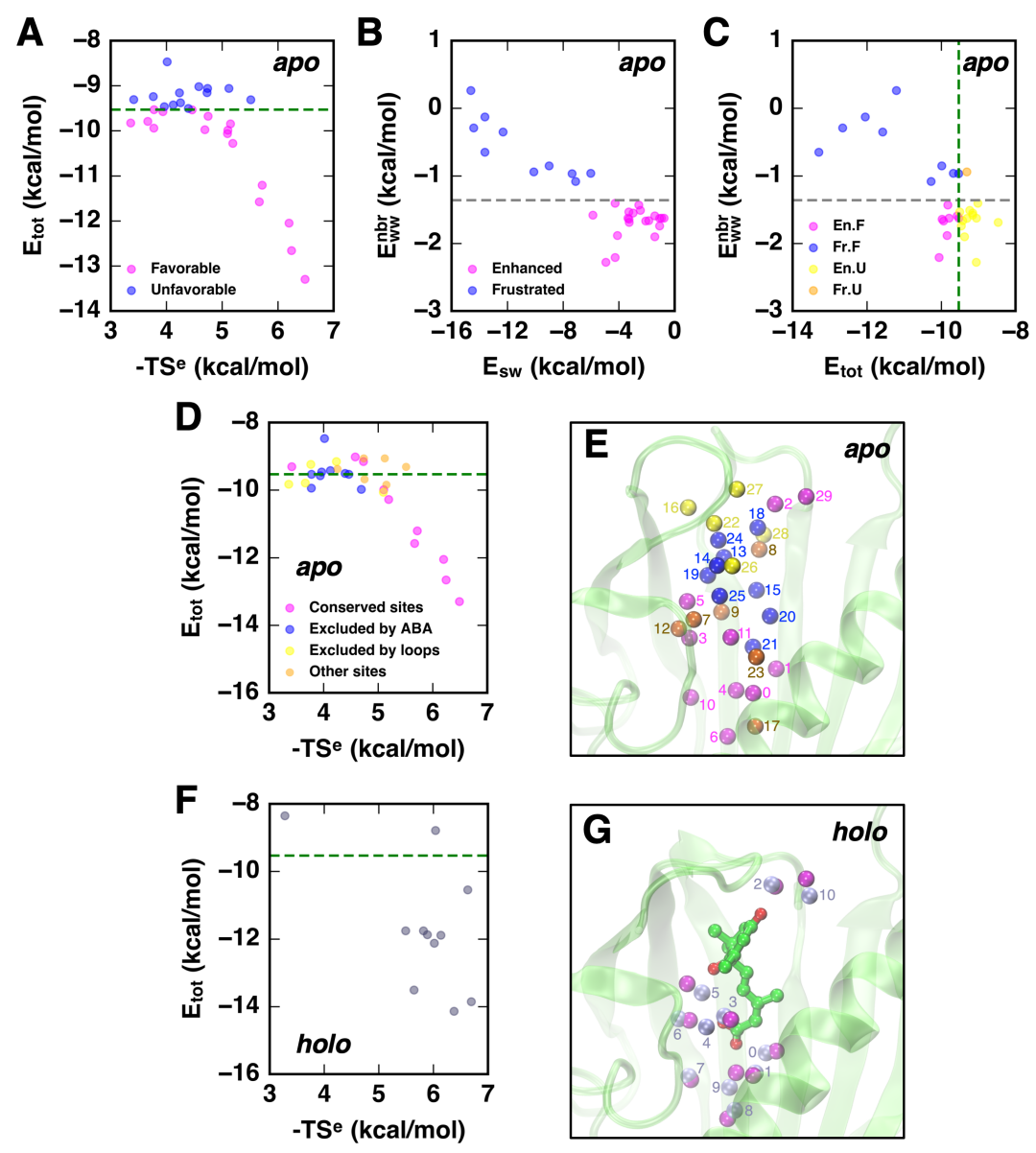

Fig. 3 Solvation structural and thermodynamic properties of the binding site in PYL2. (A) System interaction energies $\left(E_{t o t}\right)$ and excess entropic contribution $\left(-T S^{e}\right)$ to free energy, (B) solute-water $\left(E_{s w}\right)$ and per-neighbor water-water $\left(E_{w w}^{n b r}\right)$ interaction energies, and (C) $E_{t o t}$ and $E_{w w}^{n b r}$ of water molecules in the hydration sites of the apo binding cavity. (D) Hydration sites in apo PYL2 are colored according to their exclusion states upon ABA binding and PYL2 activation and (E) their locations in pocket. (F) $E_{t o t}$ and $-T S^{e}$ for the hydration sites in holo PYL2 and (G) overlay of the conserved hydration sites in apo (magenta) and holo (iceblue) PYL2. The green line and grey line indicate $E_{t o t}$ and $E_{w w}^{n b r}$ for the bulk water.

located at bottom of the pocket (Fig. 3D, E). To exclude these hydration sites, it would cause significantly large penalty for binding. The hydration site 11 at bottom and two other sites 2 and 29 at top of the binding pocket are energetically slightly unfavorable but less entropically unstable (Fig. 3D). For all the holo hydration sites excluding the two sites at top of the pocket, $E_{t o t}$ is significantly lower compared to the bulk (Fig. $3 F, G)$. Water molecules in these sites mediate the PYL2-ABA interactions and $E_{s w}$ dominantly contribute to $E_{t o t}$. Compared to the apo hydration sites, all these sites are energetically stabilized by the binding of ABA (Table S6). Meanwhile, the binding of ABA results in slight entropy loss for water molecules in these sites (except for holo sites 8 and 9). For the two holo sites 2 and 10 at top of the pocket, they are energetically destablized due to unfavored water-water interactions after ABA binds. The hydration site 2 is critical in bridging water-mediated interaction between PYL2 and PP2C, 23 and the binding of PP2C may further decrease $E_{t o t}$ of the site 2 . The hydration site 10 is excluded upon PP2C binding. The net enthalpic contribution of restructuring the conserved 10 hydration sites to free energy of binding is -9.5 $\mathrm{kcal} / \mathrm{mol}$, and the net entropic loss is $+4.05 \mathrm{kcal} / \mathrm{mol}$. Overall, these results suggest that the reorganization of water molecules in the binding site after ABA binding lead to further enthalpic contribution to binding free energy.

The solvation structural and thermodynamic data of PYL2 receptor may be utilized to understand the binding mechanism of selective ABA agonists. For example, previous crystallographic studies showed that the ABA agonist pyrabactin binds to PYL2 in a nonproductive pose which is different from the productive binding in PYL1.6061 We show that this observation can be explained by the relative penalty for exclusion of water molecules from the hydration sites (Fig. S2C-F). Specifically, the productive pose of pyrabactin would exclude water molecules in 8 apo hydration sites, including the sites 5 and 7 that are energetically favorable. In contrast, the non-productive binding would only exclude water molecules from 5 apo hydration sites, and they are relatively less stable. Thus, pyrabactin binds to PYL2 preferentially in a non-productive binding pose. Taken together, our hydration site analysis elucidates the solvation thermodynamics and structure 
bioRxiv preprint doi: https://doi.org/10.1101/721761; this version posted August 1, 2019. The copyright holder for this preprint (which was not certified by peer review) is the author/funder, who has granted bioRxiv a license to display the preprint in perpetuity. It is made available under aCC-BY-NC-ND 4.0 International license.

Table 1 Calculated thermodynamic $(\mathrm{kcal} / \mathrm{mol})$ and structural quantities for the 9 excluded hydration sites in apo PYL2 receptor by ABA upon binding, identified by clustering the solvent density distribution inside the binding site. 'Neat' represents the pure TIP3P water.

\begin{tabular}{|c|c|c|c|c|c|c|c|c|c|c|c|c|c|}
\hline index & type & $\overline{f_{o}}$ & $E_{s w}$ & $\overline{E_{w w}}$ & $E_{t o t}$ & $E_{w w}^{n b r}$ & $-T S^{e}$ & $N_{n b r}$ & $f_{\text {enc }}$ & $N_{s w}^{H B}$ & $N_{w w}^{H B}$ & $f_{w w}^{H B}$ & $N_{w w, l o s t}^{H B}$ \\
\hline neat ${ }^{59}$ & - & - & 0.00 & -9.53 & -9.53 & -1.36 & 0.00 & 5.26 & 0.00 & 0.00 & 3.33 & 0.63 & 0.00 \\
\hline 13 & En.U & 0.52 & -3.29 & -6.21 & -9.50 & -1.53 & 4.39 & 3.39 & 0.35 & 0.84 & 2.36 & 0.69 & 0.97 \\
\hline 14 & En.F & 0.53 & -3.28 & -6.70 & -9.98 & -1.64 & 4.69 & 3.21 & 0.39 & 0.93 & 2.39 & 0.74 & 0.94 \\
\hline 15 & En.U & 0.50 & -1.86 & -7.57 & -9.42 & -1.66 & 4.12 & 3.73 & 0.29 & 0.00 & 2.64 & 0.71 & 0.69 \\
\hline 18 & En.F & 0.48 & -1.42 & -8.15 & -9.57 & -1.59 & 3.93 & 4.36 & 0.17 & 0.01 & 3.20 & 0.73 & 0.13 \\
\hline 19 & En.F & 0.40 & -0.76 & -8.77 & -9.53 & -1.62 & 3.78 & 4.45 & 0.15 & 0.02 & 3.22 & 0.72 & 0.11 \\
\hline 20 & En.U & 0.39 & -3.27 & -5.20 & -8.47 & -1.69 & 4.01 & 2.65 & 0.50 & 0.59 & 1.96 & 0.74 & 1.37 \\
\hline 21 & Fr.F & 0.38 & -7.36 & -2.18 & -9.53 & -0.97 & 4.46 & 3.45 & 0.34 & 0.76 & 2.05 & 0.60 & 1.28 \\
\hline 24 & En.F & 0.34 & -2.08 & -7.86 & -9.94 & -1.67 & 3.77 & 4.01 & 0.24 & 0.30 & 3.01 & 0.75 & 0.32 \\
\hline 25 & En.U & 0.33 & -1.08 & -8.39 & -9.46 & -1.74 & 3.96 & 4.08 & 0.23 & 0.00 & 3.05 & 0.75 & 0.28 \\
\hline
\end{tabular}

in PYL2 and suggest that the displacement and reorganization of water molecules in the binding site lead to entropic and enthalpic contributions to binding free energy, respectively.

\section{Displacement of water molecules in the binding site causes free energy barrier to ABA binding.}

The free energy barrier to ABA binding is associated with the transition from state 3 to state 4 in both monomeric and dimeric receptors (Fig. 1A, B), which appears to be caused by exclusion of water molecules from the binding site 32 . Fig. 4 A, B show that substantial exclusions of water molecules from ABA solvation shell (within $5 \AA$ of $\mathrm{ABA}$ ) and the binding site are associated with $\mathrm{ABA}$ binding in both receptors. For the dimeric receptor, the number of waters that solvate ABA decreases from $\sim 50$ to $\sim 20$ after ABA enters into the pocket, in contrast to the slow decrease in the monomeric receptor. The explanation is that after ABA enters into the binding cavity, ABA is fully enclosed by both protomers in the dimeric PYL2, whereas ABA remains largely exposed to the solvent in the monomeric PYL2. This can contribute to the relatively higher stability of the intermediate states in the dimeric PYL2 receptor, such as the state 3. In terms of the water molecules in the pocket, we observe the steady decreases along the binding process in both monomeric and dimeric receptors. Especially, there are $\sim 3-4$ water molecules being excluded from the pocket from the state 3 to the state 4 (Fig. 4A, B). The observed binding free energy barriers appear to be associated with the penalty for exclusion of these water molecules.

We further seek to understand the exclusion of water molecules in apo hydration sites along ABA binding process. We first align the structures of the binding intermediate states (state 2 and state 3 ) in dimeric PYL2 to the inactive structure. Then we determine the exclusion of hydration sites in these binding intermediate states (Fig. 4C, D). Interestingly, ABA does not overlap with any hydration sites in the state 2 suggesting that no water molecules in hydration sites have been excluded by ABA. Several hydration sites (indices $2,18,24,27,29$ ) are excluded by S89 in the gate loop and H119 and R120 in the latch loop. This suggests that there is little penalty for the transition from the unbound state to the intermediate state 2 (Fig. 13). In state 3 , the carboxylate group of ABA moves toward the binding site and excludes water molecule in the hydration site 20 . The penalty for excluding hy- dration site 20 is low since it has the highest energy within all hydration sites. Hydration sites 13 and 18 are excluded by H119 in the latch loop. For ABA to move from state 3 to the bound state, the water molecules in the six other hydration sites (14, $15,19,21,24,25)$ remain to be excluded by ABA. Notably, these sites have nearly comparable $(15,19,21,25)$ and even lower (sites 14, 24) $E_{\text {tot }}$ compared to the bulk water molecules. Exclusion of these water molecules would introduce significant penalty for ABA binding. Furthermore, several of these hydration sites to be excluded are buried below ABA and can not be easily expelled into the bulk. As a result, the exclusion of these water molecules lead to the accumulative free energy barrier to ABA binding (Fig. 13). Overall, our results suggest that ABA binds to PYL2 via a pathway with the minimal cost for exclusion of water molecules and the exclusion of water molecules result in the free energy barrier to ABA binding.
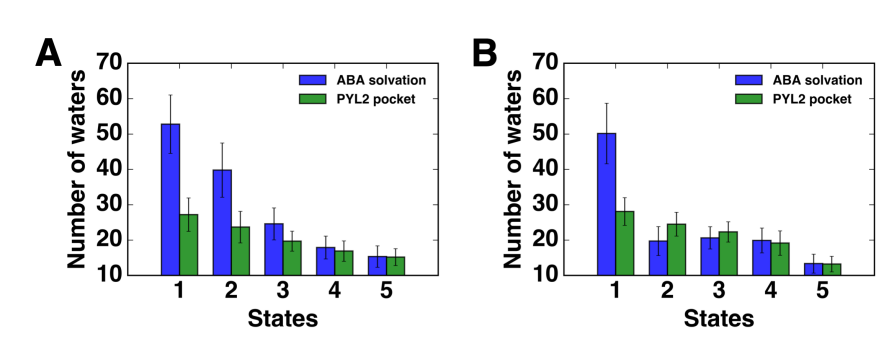

C
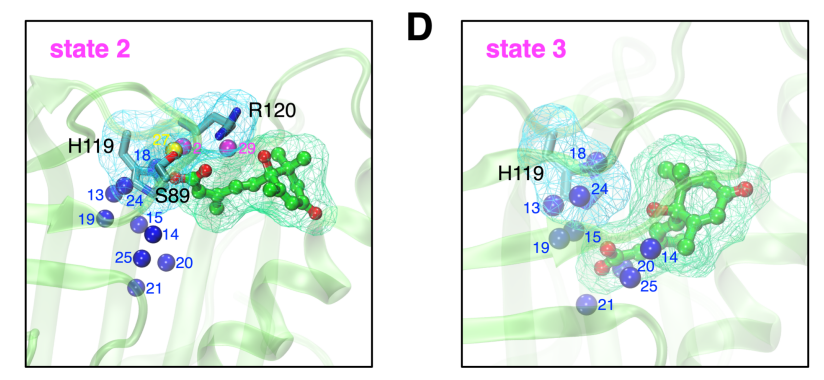

Fig. 4 Exclusion of water molecules during $A B A$ binding. The numbers of water molecules in $\mathrm{ABA}$ solvation shell $(<5 \AA$ of $\mathrm{ABA}$, blue) and the binding pocket (green) for the states $1-5$ in $(A)$ the monomeric and $(B)$ the dimeric PYL2 receptors identified from TPT. The snapshots show $(H)$ the exclusion of hydration sites in the binding $(C)$ intermediate state 3 and (D) intermediate state 4 . 

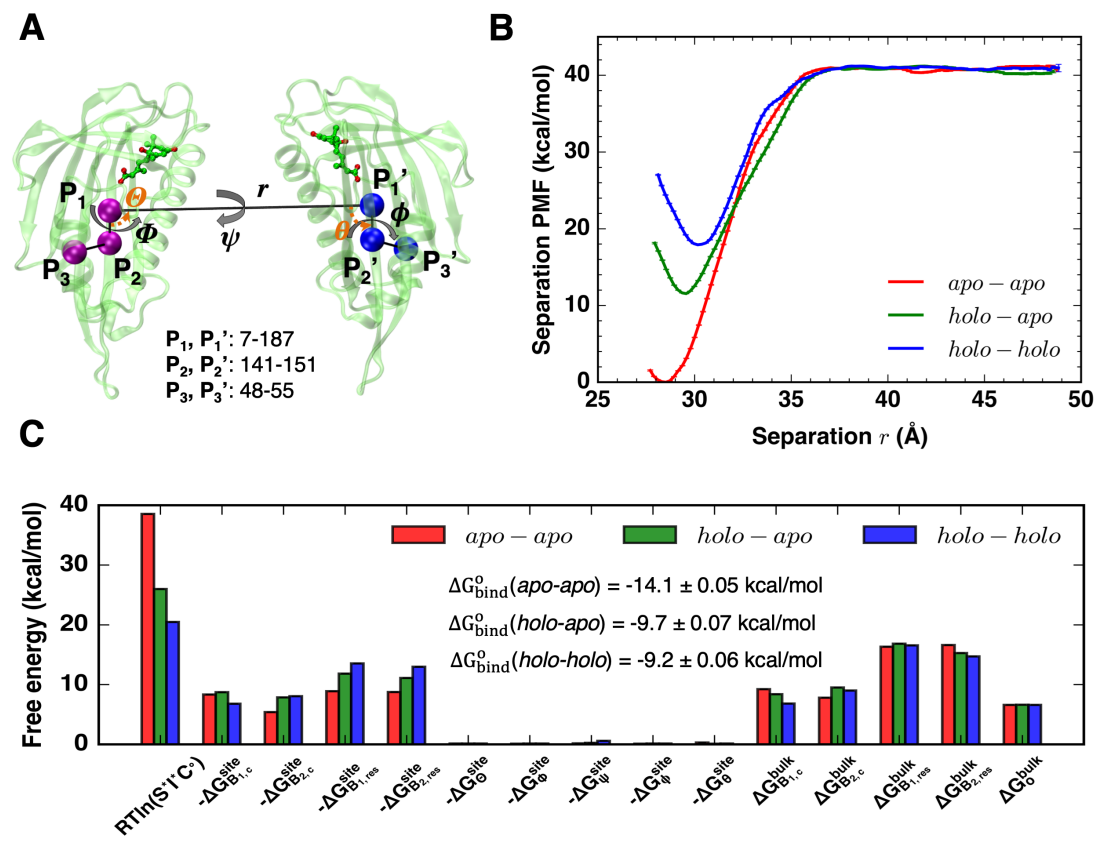

Fig. 5 Determination of PYL2 homodimeric association free energies. (A) Snapshot of the holo-holo PYL2 and the collective variables used in PYL2 separation REUS simulations. The center of mass distance between the two protomers $r\left(\mathrm{P}_{1}-\mathrm{P}_{1}^{\prime}\right.$ distance), and the Euler angles $\phi\left(\mathrm{P}_{1}-\mathrm{P}_{1}^{\prime}-\mathrm{P}_{2}^{\prime}-\mathrm{P}_{3}^{\prime}\right)$ and $\theta$ $\left(P_{1}-P_{1}^{\prime}-P_{2}^{\prime}\right)$ defines the relative position of the two protomers. The Euler angles, $\Theta\left(P_{1}^{\prime}-P_{1}-P_{2}\right), \Phi\left(P_{1}^{\prime}-P_{1}-P_{2}-P_{3}\right)$, and $\psi\left(P_{2}^{\prime}-P_{1}^{\prime}-P_{1}-P_{2}\right)$, relate the relative orientation between the two protomers. In addition, PYL2 conformations are restrainted by RMSDs of the two protomers $\left(B_{1, c}, B_{2, c}\right)$ and the interfacial residues $\left(B_{1, \text { res }}, B_{2, \text { res }}\right)$ from the initial strutcures. (B) Potential of mean force (PMF) profiles for the separations of the apo-apo (red), the holo-apo (green), and the holo-holo (blue) PYL2. The error bars on the PMFs are shown. (C) Free energies associated to the components of $\Delta G_{b i n d}^{\circ}$ for the apo-apo (red), the holo-apo (green) and the holo-holo (blue) PYL2 complexes. The error bars for all components are less than $0.04 \mathrm{kcal} / \mathrm{mol}$.

\section{Binding of ABA and subsequent closure of the gate loop desta- bilizes PYL2 complex.}

To quantitatively characterize how ABA changes the stability of PYL2 complex, we seek to determine how standard PYL2 association free energy $\left(\Delta G_{b i n d}^{\circ}\right)$ changes before and after ABA binding. We compare $\Delta G_{\text {bind }}^{\circ}$ for the apo-apo, the holo-apo, and the holo-holo PYL2 complexes, where apo denotes an inactive protomer without ABA and holo denotes an active protomer with ABA bound. We employ potential of mean force (PMF)-based method for accurate estimation of standard protein-protein binding free energy. 49 In each case, we separate two protomers along a vector $r$ that connects the center of mass of two protomers in the presence of a series of geometrical restraints (Fig. 54A). To determine the separation PMF, we select a range of complex conformations with $r$ evenly distributed in a certain range from the associated state to the fully dissociate state. Each conformation is used to seed a new replica simulation, with a harmonic potential acting on $r$ to restrain the distance between two protomers. From these simulations, the separation PMF can be estimated via a well-established statistical free energy method, multistate Bennett acceptance ratio (MBAR). 48 The geometrical restraints are used to accelerate the convergence of separation PMF, which include the conformational restraints $\left(B_{1, c}, B_{2, c}\right.$, $\left.B_{1, \text { res }}, B_{2, \text { res }}\right)$ and the restraints on the relative positions $(\phi, \theta)$ and orientations $(\Theta, \Phi$ and $\psi$ ) between two protomers (Fig. 5.A). The contributions of separation PMF and all restraints to $\Delta G_{\text {bind }}^{\circ}$ can be determined individually (Supplementary Methods).
The starting structures of the holo-apo, and the holo-holo PYL2 complexes are obtained via short targeted MD simulations. We select 41 windows for each complex, with the distance $r$ evenly distributed between 28-48 A. We perform 8 ns replica exchange umbrella sampling (REUS) MD simulations for each replica, and use MBAR to determine the PMFs $W(r)$. Fig. S3 shows that the separation PMFs for all three receptors are converged within 8 ns/window simulations. The separation PMF profiles for the apo-apo, the holo-apo, and the holo-holo PYL2 complexes are given in Fig. 5B , along with the associated error bars. The wells in the three PMFs between 28-31 $\AA$ correspond to the associated states. $W(r)$ converges to constant when $r$ is greater than $38 \AA$, showing that the two protomers are fully separated. The well depths for the apo-apo, the holo-apo, and the holo-holo are $41 \mathrm{kcal} / \mathrm{mol}, 30 \mathrm{kcal} / \mathrm{mol}$ and $23 \mathrm{kcal} / \mathrm{mol}$ and their related contributions to $\Delta G_{\text {bind }}^{\circ}$ are $-38.5 \mathrm{kcal} / \mathrm{mol},-26.0$ $\mathrm{kcal} / \mathrm{mol},-20.5 \mathrm{kcal} / \mathrm{mol}$. It should be noted that these energy contributions include the effects of numerous restraints applied during the separation REUS MD simulations. However, these results qualitatively suggest that the stability of PYL2 complex decreases upon ABA binding. Also, we can observe that the minimum of $W(r)$ slightly increases from $28.5 \AA$ to $30 \AA$.

The contributions of adding the geometrical restraints in the associated state (denoted by site) and removing these restraints in the fully separated state (denoted by bulk) are calculated by determining PMFs for each restraints (Supplementary Methods). 
A

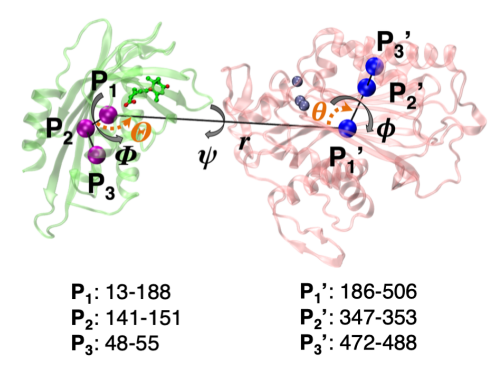

C

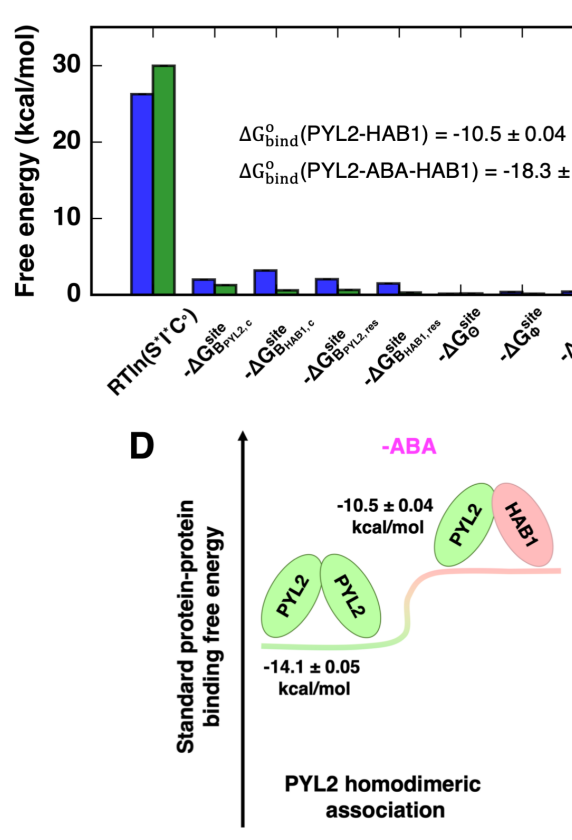

B

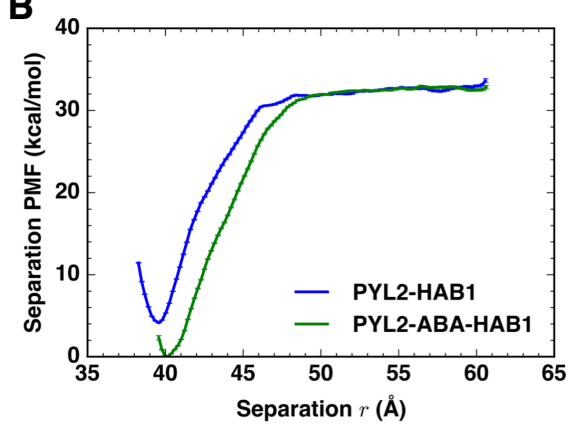


tively show that the binding of ABA destabilizes PYL2 complex by shifting the conformation equilibrium, while PYL2 remains stable after ABA binding.

\section{Presence of ABA substantially stabilizes PYL2-HAB1 complex and further promotes PYL2 dissociation.}

In the presence of both $\mathrm{ABA}$ and PP2C, the ABA-bound PYL2 interact with both PYL2 to form a stable homodimer and PP2C to form a heterodimer. To understand the competitive binding mechanism between PYL2 homodimeric association and PYL2PP2C heterodimeric association, we seek to determine $\Delta G_{\text {bind }}^{\circ}$ for PYL2-HAB1 association. We determine $\Delta G_{\text {bind }}^{\circ}$ for both the PYL2-HAB1 (in the absence of ABA) and PYL2-ABA-HAB1 (in the presence of ABA) complexes. By comparing with $\Delta G_{\text {bind }}^{\circ}$ for the apo-apo, the holo-apo, and the holo-holo PYL2, we aim to establish a thermodynamic model for competitive association between PYL2 homodimerization and PYL2-HAB1 heterodimerization. In addition, crystallographic studies have shown that PP2C form water-mediated interaction with $\mathrm{ABA}$, and PP2C is therefore widely considered as a ABA co-receptor. 31 Biologically, it is also interesting to investigate how much the presence of $\mathrm{ABA}$ can stabilize the PYL2-HAB1 complex.

Using a similar protocol as in PYL2 separation, the separation PMFs for the PYL2-HAB1 and the PYL2-ABA-HAB1 complexes were determined in the presence of conformational ( $B_{P Y L 2, c}$, $\left.B_{H A B 1, c}, B_{P Y L 2, \text { res }}, B_{H A B 1, \text { res }}\right)$ and angular restraints $(\Theta, \Phi, \psi, \phi$, $\theta$ ) (Fig. 6A). The separation PMFs converge with $8 \mathrm{~ns} /$ window REUS MD simulations (Fig. S5A, B). The wells in the two PMFs between 39-41 $\AA$ correspond to the associated PYL2-HAB1 and PYL2-ABA-HAB1 complexes (Fig. 6B). $W(r)$ converges when $r$ is greater than $50 \AA$, showing that PYL2 and HAB1 are fully separated. The well depths for the PYL2-HAB1 and the PYL2-ABA-HAB1 are $28 \mathrm{kcal} / \mathrm{mol}$ and $33 \mathrm{kcal} / \mathrm{mol}$, and their contributions to $\Delta G_{\text {bind }}^{\circ}$ are $-26.3 \mathrm{kcal} / \mathrm{mol}$ and $-30.0 \mathrm{kcal} / \mathrm{mol}$, respectively. These results suggest that the presence of $A B A$ in the active PYL2 stabilizes the binding with HAB1. The contributions of adding the geometrical restraints in the site and removing these restraints in the bulk are calculated by determining PMFs for each restraints (Fig. S5C, D). From these PMFs, we determine the contributions of all restraints to $\Delta G_{\text {bind }}^{\circ}$ (Fig. 6r).

We obtain $\Delta G_{\text {bind }}^{\circ}$ for the PYL2-HAB1 and the PYL2-ABA-HAB1 complexes by summing over all individual contributions to $\Delta G_{\text {bind }}^{\circ}$, which are $-10.5 \mathrm{kcal} / \mathrm{mol}$ and $-18.3 \mathrm{kcal} / \mathrm{mol}$ respectively. Our free energy results show that the presence of $A B A$ in PYL2 substantially increases the thermostability of PYL2-HAB1 complex by $-7.8 \mathrm{kcal} / \mathrm{mol}$. The ABA's stabilizing effects may be attributed to two aspects. First, the water-mediated interaction between ABA and HAB1 is expected to result in stronger association between PYL2 and HAB1. Second, the binding of ABA may perturb the conformational entropy of PYL2 receptor in the bulk and site, thereby contributing to the PYL2-HAB1 binding free energy. Overall, our results suggest that $\mathrm{ABA}$ is essential in stabilizing PYL2-HAB1 interactions.
Take together the free energy results for PYL2 homodimeric association, we can obtain a complete thermodynamic model to understand the competitive interactions between PYL2 selfassociation and PYL2-HAB1 heterodimeric association (Fig. 6P). In the absence of ABA, the $\Delta G_{\text {bind }}^{\circ}$ for the apo-apo PYL2 and the PYL2-HAB1 complex are $-14.1 \mathrm{kcal} / \mathrm{mol}$ and $-10.5 \mathrm{kcal} / \mathrm{mol}$, respectively. The PYL2 homodimeric association is favored compared to the heterodimeric association between PYL2 and PP2C. In the presence of ABA, the PYL2 complex is destabilized and the PYL2-HAB1 complex is stabilized by the binding of ABA. The $\Delta G_{\text {bind }}^{\circ}$ for the holo-holo PYL2 and the PYL2-ABA-HAB1 complex are $-9.2 \mathrm{kcal} / \mathrm{mol}$ and $-18.3 \mathrm{kcal} / \mathrm{mol}$. As a result, the heterodimeric association between PYL2 and HAB1 is largely favored than the PYL2 homodimeric association. Overall, our results suggest that the presence of ABA stabilizes PYL2-HAB1 complex and further promotes PYL2 dissociation.

\section{Conclusions}

The dimeric PYR/PYL/RCAR receptors play an important role in a variety of $A B A$ responses,, $16 \mid 18$ while fundamental understanding of their activation mechanism remains incomplete. In particular, the dynamic and energetic basis of ABA-induced activation and dissociation of dimeric receptors is lacking. In addition, the role of water networks in the receptor binding site on ABA binding has never been quantitatively examined despite its importance in protein-ligand binding. Our MD study investigates the atomic scale dynamics of ABA perception by PYL2 in both its monomeric and dimeric forms, allowing for a detailed understanding of how dimerization affects receptor activation process. Using the HSA method, we elucidate the solvation structural and thermodynamic properties of the binding site, and provide quantitative understanding how water molecules contribute to ligand binding affinity and penalty. From standard protein-protein binding free energy calculations, we quantitatively characterize how ABA changes the thermodynamic stability of both PYL2 homodimer and PYL2-HAB1 heterodimer, thereby gaining energetic insights into PYL2 dissociation and PYL2-HAB1 association processes.

On ABA perception, our MD simulations have uncovered the key intermediate states involved in the binding of $\mathrm{ABA}$ and subsequent activation of both monomeric and dimeric PYL2 (Fig. 1 and Fig. S1). The key residues that mediate PYL2-ABA interactions in our predicted intermediate states have been shown to be critical for ABA receptor activity from previous mutation studies.23 Free energy landscapes suggest that PYL2 complex substantially constrains the conformational plasticity of the gate loop, thereby stabilizing the open conformational state and destabilizing the closed state (Fig. 19, B). Furthermore, the PYL2 complex stabilizes the intermediate state due to the enhanced hydrophobic interactions. As a result, the ABA off-binding rate increases due to dimerization (Fig. 2), leading to lower ABA affinity in the dimeric PYL2. On receptor activation, our results suggest that $\mathrm{ABA}$ is required to trigger the gate loop closure for the dimeric PYL2, and the gate loop remains in open conformation even after ABA binding (Fig. 22), in agreement with 
HDX experimental data. ${ }^{55}$ In this sense, ABA binding is necessary but far from sufficient condition for the activation of the dimeric PYL2. Overall, our simulations have explained the molecular basis of intrinsic lower $\mathrm{ABA}$ sensitivity for dimeric $\mathrm{ABA}$ receptors.

On the effect of water thermodynamics on ABA perception, our HSA calculations show that the displacement and reorganization of water molecules in the binding site contribute to free energy of ABA binding (Fig. 3). Furthermore, the displacement of water molecules causes the major free energy barrier to ABA binding (Fig. 4). The overall thermodynamic effects of water molecules on $\mathrm{ABA}$ perception can be understood via enthalpy-entropy compensation during the binding process. The gain of entropy by expelling water molecules from the 9 apo hydration sites dominantly contributes to ABA affinity, whereas the gain of enthalpy is relatively small. Despite the fact that all the apo hydration sites are entropically unstable, displacement of more water molecules in the binding site, especially any of those which are energetically stable, would lead to significant increase of enthalpic penalty for ABA binding. This may partially explain the non-productive binding pose of pyrabactin in PYL2 (Fig. S2), which serves as an ABA agonist for PYL1 but an ABA antagonist for PYL2. ${ }^{60161}$ After ABA binding, water molecules in the holo hydration sites become energetically stabilized but entropically slightly destabilized relative to the apo states. As a consequence, the reorganization of water network further contributes to free energy of binding. On the other hand, our results have shown that PYL2 solvation structure and thermodynamics affects not only the binding mode and affinity of ABA but also the binding process of $A B A$. ABA binds to PYL2 in a pathway which has the minimal cost for displacing water molecules in apo hydration sites. Overall, our HSA calculations provide insights into the role of water networks in the binding site on ABA perception, which can potentially be exploited for virtual screening and rational design of selective ABA agonists and antagonists. ${ }^{62}$

On PYL2 dissociation, our free energy calculation results suggest that the activation of both protomers introduce $\sim 4.9 \mathrm{kcal} / \mathrm{mol}$ differences in the stability of PYL2 complex (Fig. 5). Interestingly, our results show that the activation of the first protomer in the apo-apo PYL2 contributes mostly to the changes in association free energy $(\sim 4.4 \mathrm{kcal} / \mathrm{mol})$, while the activation of the second protomer only cause slight additional changes $(\sim 0.5 \mathrm{kcal} / \mathrm{mol})$. However, both the holo-apo and the holo-holo complexes are highly stable, with free energies of $-9.7 \mathrm{kcal} / \mathrm{mol}$ and -9.2 $\mathrm{kcal} / \mathrm{mol}$, respectively. The binding of ABA can only slightly shift the equilibrium of PYL2 complex formation. The PYL2 dissociation is further promoted by the presence of PP2C in solution. It should be noted that ABA-bound PYL2 has strong interaction with PP2Cs in solution, with a dissociation constant $K_{d}$ between $150-250 \mathrm{nM} \cdot{ }^{23 \mid 26}$ Our free energy calculation results show that the presence of ABA substantially decrease PYL2-HAB1 association free energy from $-10.5 \mathrm{kcal} / \mathrm{mol}$ to $-18.3 \mathrm{kcal} / \mathrm{mol}$ (Fig. 6). Therefore, the presence of ABA makes the homodimeric PYL2 association less competitive than the heterodimeric PYL2PP2Cs association as compared to that in the absence of ABA.
Overall, our free energy calculations establish the thermodynamic model for explaining the competitive associations between PYL2 homodimerization and PYL2-HAB1 heterodimerization (Fig. 6p). From a broad perspective, computational methods for studying protein-protein association can be useful in understanding the combinatorial interactions between core components (PYR/PYL/RCAR, PP2Cs, SnRK2s) in ABA signaling.

In Arabidopsis thaliana, PYL2, PYL5 and PYL10 receptors belong to three individual classes of PYR/PYL/RCAR receptors based on their sequence similarities. ${ }^{[16}$ Combining the results from our recent work on the PYL5 and PYL10 receptors,, 32 we can draw several conclusions regarding the similarities and the differences in activation of the three classes of ABA receptors. On the similarities, (1) ABA binding is necessary but not sufficient for full activation of PYR/PYL/RCAR receptors. We have shown that the binding of $\mathrm{ABA}$ can shift the conformational equilibrium of the gate loop, thereby increasing the equilibrium probability of closed gate loop conformations. However, the gate loop remains flexible between open and closed conformations. 55 Strikingly, the dimerization of PYR/PYL/RCAR receptors substantially favor the open gate loop conformations, due to the fact that the gate loop is involved in forming the dimer interface. PP2C binding is required to stabilize the closed gate loop conformations for full receptor activation. In the case of dimeric receptors, PP2C might be required for their full dissociation. (2) A large binding free energy barrier $(\sim 4-5 \mathrm{kcal} / \mathrm{mol})$ is observed for all the receptors, which is associated with the exclusion of water molecules from the binding pocket. The displacement and reorganization of water networks in the binding site contribute to overall free energy of ABA binding. These computational results highlight the role of water molecules in ABA perception.

On the differences among three clades of ABA receptors in Arabidopsis thaliana, (1) the ABA binding pathways and the relative stability of transition states in the pathways vary depending on the sequence and the oligomeric state. While the key polar residues that mediate $\mathrm{ABA}$ binding are conserved, the binding pathways are not conserved across ABA receptors. For example, we previous reported that the side chain switch of F58 in the PYL10 is required for ABA binding, 32 whereas this is not the same in both PYL2 and PYL5 receptors. In this study, we have shown that PYL2 complex stabilizes the binding intermediate state while destabilizes the ABA-bound, close gate loop state. It is possible that the variations in solvation structural and thermodynamic properties across different ABA receptors due to amino acid substitutions contribute to the binding differences. (2) ABA-independent and ABA-dependent activation depends on the sequence and the oligomeric state. For the monomeric receptors, we have shown that the gate loop has a higher degree of conformational plasticity, which results in the experimentally observed ABA-independent activity of these receptors. Notably, the PYL10 has shown a distinct constitutive activity. ${ }^{2729}$ For the dimeric receptors, $\mathrm{ABA}$ binding is required to trigger receptor activity because the dimer conformation cannot be broken by PP2Cs in the absence of ABA. 
Finally, our computational studies have provided physical insights into the structural, dynamic and energetic basis of the activation of ABA receptors in Arabidopsis thaliana, which are challenging to obtain with experimental approaches. Beyond Arabidopsis thaliana, there are a growing number of studies on ABA receptors in higher plants, such as rice $\frac{63}{63}$, soybean $\sqrt{64}$ and maize 65 . However, the structural information on ABA receptors in higher plants is still scarce. Our key findings on the activation mechanism of ABA receptors could be generalized across other plant species given that several core components in ABA signaling and the sequences of $\mathrm{ABA}$ receptors are highly conserved. On the other hand, our computational modeling framework can be extended to understand ABA signaling mechanism in higher plants. In addition to understanding the molecular basis, computational chemistry approaches could be useful in rational engineering of $A B A$ receptors and design of new agrochemicals to improve chemical and genetic control of ABA responses.

\section{Conflicts of interest}

There are no conflicts to declare.

\section{Acknowledgements}

The authors acknowledge the support from the Blue Waters sustained-petascale computing project, which is funded by the National Science Foundation (awards OCI-0725070 and ACI1238993) and the state of Illinois. D.S. acknowledges the support from Foundation for Food and Agriculture Research via the New Innovator Award in Food \& Agriculture Research. C.Z. acknowledges the support by 3M Corporate Fellowship from the University of Illinois. The authors thank Alexander S. Moffett from the University of Illinois for the helpful discussions on standard protein-protein binding free energy calculations.

\section{Author contributions}

C.Z. and D.S. designed the project. C.Z. performed the simulations, analyzed the data, and wrote the manuscript with input from D.S.

\section{References}

1 J.-K. Zhu, Cell, 2016, 167, 313-324.

2 K. Vishwakarma, N. Upadhyay, N. Kumar, G. Yadav, J. Singh, R. K. Mishra, V. Kumar, R. Verma, R. G. Upadhyay, M. Pandey and S. Sharma, Front. Plant Sci., 2017, 8, 161.

3 L. Xiong and J.-K. Zhu, Plant Physiol., 2003, 133, 29-36.

4 S. Munemasa, F. Hauser, J. Park, R. Waadt, B. Brandt and J. I. Schroeder, Curr. Opin. Plant Biol., 2015, 28, 154-162.

5 S. R. Cutler, P. L. Rodriguez, R. R. Finkelstein and S. R. Abrams, Annu. Rev. Plant Biol., 2010, 61, 651-679.

6 T. Umezawa, N. Sugiyama, M. Mizoguchi, S. Hayashi, F. Myouga, K. Yamaguchi-Shinozaki, Y. Ishihama, T. Hirayama and K. Shinozaki, Proc. Natl. Acad. Sci. U.S.A., 2009, 106, 1758817593.

7 F.-F. Soon, L.-M. Ng, X. E. Zhou, G. M. West, A. Kovach, M. H. E. Tan, K. M. Suino-Powell, Y. He, Y. Xu, M. J. Chalmers, J. S. Brunzelle, H. Zhang, H. Yang, H. Jiang, J. Li, E.-L. Yong,
S. Cutler, J.-K. Zhu, P. R. Griffin, K. Melcher and H. E. Xu, Science, 2011, 335, 85-88.

8 S.-Y. Park, P. Fung, N. Nishimura, D. R. Jensen, H. Fujii, Y. Zhao, S. Lumba, J. Santiago, A. Rodrigues, T.-F. Chow, S. E. Alfred, D. Bonetta, R. Finkelstein, N. J. Provart, D. Desveaux, P. L. Rodriguez, P. McCourt, J.-K. Zhu, J. I. Schroeder, B. F. Volkman and S. R. Cutler, Science, 2009, 324, 1068-1071.

9 H. Fujii, P. E. Verslues and J.-K. Zhu, Proc. Natl. Acad. Sci. U.S.A., 2011, 108, 1717-1722.

10 D. Geiger, S. Scherzer, P. Mumm, A. Stange, I. Marten, H. Bauer, P. Ache, S. Matschi, A. Liese, K. A. S. Al-Rasheid, T. Romeis and R. Hedrich, Proc. Natl. Acad. Sci. U.S.A., 2009, 106, 21425-21430.

11 S. C. Lee, W. Lan, B. B. Buchanan and S. Luan, Proc. Natl. Acad. Sci. U.S.A., 2009, 106, 21419-21424.

12 J. D. Helander, A. S. Vaidya and S. R. Cutler, Bioorg. Med. Chem., 2016, 24, 493-500.

13 Z. Yang, J. Liu, S. V. Tischer, A. Christmann, W. Windisch, H. Schnyder and E. Grill, Proc. Natl. Acad. Sci. U.S.A., 2016, 113, 6791-6796.

14 Z. Yang, J. Liu, F. Poree, R. Schaeufele, H. Helmke, J. Frackenpohl, S. Lehr, P. von Koskull-Döring, A. Christmann, H. Schnyder, U. Schmidhalter and E. Grill, Plant Physiology, 2019, pp.01238.2018.

15 R. Mega, F. Abe, J.-S. Kim, Y. Tsuboi, K. Tanaka, H. Kobayashi, Y. Sakata, K. Hanada, H. Tsujimoto, J. Kikuchi, S. R. Cutler and M. Okamoto, Nat. Plants, 2019, 5, 153-159.

16 M. Okamoto, F. C. Peterson, A. Defries, S.-Y. Park, A. Endo, E. Nambara, B. F. Volkman and S. R. Cutler, Proc. Natl. Acad. Sci. U.S.A., 2013, 110, 12132-12137.

17 M. Cao, X. Liu, Y. Zhang, X. Xue, X. E. Zhou, K. Melcher, P. Gao, F. Wang, L. Zeng, Y. Zhao, Y. Zhao, P. Deng, D. Zhong, J.-K. Zhu, H. E. Xu and Y. Xu, Cell Res., 2013, 23, 1043-1054.

18 A. S. Vaidya, F. C. Peterson, D. Yarmolinsky, E. Merilo, I. Verstraeten, S.-Y. Park, D. Elzinga, A. Kaundal, J. Helander, J. Lozano-Juste, M. Otani, K. Wu, D. R. Jensen, H. Kollist, B. F. Volkman and S. R. Cutler, ACS Chem. Biol., 2017, 12, 2842-2848.

19 M.-J. Cao, Y.-L. Zhang, X. Liu, H. Huang, X. E. Zhou, W.-L. Wang, A. Zeng, C.-Z. Zhao, T. Si, J. Du, W.-W. Wu, F.-X. Wang, H. E. Xu and J.-K. Zhu, Nat. Commun., 2017, 8, 1183.

20 K. Nemoto, M. Kagawa, A. Nozawa, Y. Hasegawa, M. Hayashi, K. Imai, K. Tomii and T. Sawasaki, Sci. Rep., 2018, 8, 4268.

21 S.-Y. Park, F. C. Peterson, A. Mosquna, J. Yao, B. F. Volkman and S. R. Cutler, Nature, 2015, 520, 545.

22 D. Elzinga, E. Sternburg, D. Sabbadin, M. Bartsch, S.-Y. Park, A. Vaidya, A. Mosquna, A. Kaundal, S. Wendeborn, M. Lachia, F. V. Karginov and S. R. Cutler, ACS Chem. Biol., 2019, 14, 332-336.

23 K. Melcher, L.-M. Ng, X. E. Zhou, F.-F. Soon, Y. Xu, K. M. Suino-Powell, S.-Y. Park, J. J. Weiner, H. Fujii, V. Chinnusamy, A. Kovach, J. Li, Y. Wang, J. Li, F. C. Peterson, D. R. Jensen, E.-l. Yong, B. F. Volkman, S. R. Cutler, J.-K. Zhu and E. Xu, Nature, 2009, 462, 602. 
24 N. Nishimura, K. Hitomi, A. S. Arvai, R. P. Rambo, C. Hitomi, S. R. Cutler, J. I. Schroeder and E. D. Getzoff, Science, 2009, 326, 1373-1379.

25 J. Santiago, F. Dupeux, A. Round, R. Antoni, S.-Y. Park, M. Jamin, S. R. Cutler, P. L. Rodriguez and J. A. Márquez, Nature, 2009, 462, 665.

26 P. Yin, H. Fan, Q. Hao, X. Yuan, D. Wu, Y. Pang, C. Yan, W. Li, J. Wang and N. Yan, Nat. Struct. Mol. Biol., 2009, 16, 12301236.

27 Q. Hao, P. Yin, W. Li, L. Wang, C. Yan, Z. Lin, J. Z. Wu, J. Wang, S. F. Yan and N. Yan, Mol. Cell, 2011, 42, 662-672.

28 X. Zhang, Q. Zhang, Q. Xin, L. Yu, Z. Wang, W. Wu, L. Jiang, G. Wang, W. Tian, Z. Deng, Y. Wang, Z. Liu, J. Long, Z. Gong and Z. Chen, Structure, 2012, 20, 780-790.

29 D. Sun, H. Wang, M. Wu, J. Zang, F. Wu and C. Tian, Biochem. Biophys. Res. Commun., 2012, 418, 122-127.

30 F. Dupeux, J. Santiago, K. Betz, J. Twycross, S.-Y. Park, L. Rodriguez, M. Gonzalez-Guzman, M. R. Jensen, N. Krasnogor, M. Blackledge, M. Holdsworth, S. R. Cutler, P. L. Rodriguez and J. A. Márquez, EMBO J., 2011, 30, 4171-4184.

31 P. Rodriguez, J. Lozano-Juste and A. Albert, Adv. Bot. Res., 2019.

32 S. Shukla, C. Zhao and D. Shukla, Structure, 2019, 27, 692702.e3.

33 Y. Ma, I. Szostkiewicz, A. Korte, D. Moes, Y. Yang, A. Christmann and E. Grill, Science, 2009, 324, 1064-1068.

34 R. Abel, T. Young, R. Farid, B. J. Berne and R. A. Friesner, J. Am. Chem. Soc., 2008, 130, 2817-2831.

35 A. Biela, N. N. Nasief, M. Betz, A. Heine, D. Hangauer and G. Klebe, Angew Chem. Int. Ed., 2013, 52, 1822-1828.

36 B. Breiten, M. R. Lockett, W. Sherman, S. Fujita, M. Al-Sayah, H. Lange, C. M. Bowers, A. Heroux, G. Krilov and G. M. Whitesides, J. Am. Chem. Soc., 2013, 135, 15579-15584.

37 T. Young, R. Abel, B. Kim, B. J. Berne and R. A. Friesner, Proc. Natl. Acad. Sci. U.S.A., 2007, 104, 808-813.

38 J. M. Fox, K. Kang, M. Sastry, W. Sherman, B. Sankaran, P. H. Zwart and G. M. Whitesides, Angew Chem. Int. Ed., 2017, 56, 3833-3837.

39 S. G. Krimmer, J. Cramer, M. Betz, V. Fridh, R. Karlsson, A. Heine and G. Klebe, J. Med. Chem., 2016, 59, 1053010548.

40 S. M. Gopal, F. Klumpers, C. Herrmann and L. V. Schäfer, Phys. Chem. Chem. Phys., 2017, 19, 10753-10766.

41 K. J. Kohlhoff, D. Shukla, M. Lawrenz, G. R. Bowman, D. E. Konerding, D. Belov, R. B. Altman and V. S. Pande, Nat. Chem., 2013, 6, 15-21.

42 B. E. Husic and V. S. Pande, J. Am. Chem. Soc., 2018, 140, 2386-2396.
43 D. Shukla, C. X. Hernández, J. K. Weber and V. S. Pande, Acc. Chem. Res., 2015, 48, 414-422.

44 A. S. Moffett, K. W. Bender, S. C. Huber and D. Shukla, J. Biol. Chem., 2017, 292, 12643-12652.

45 B. Selvam, Y.-C. Yu, L.-Q. Chen and D. Shukla, ACS Cent. Sci., 2019, 5, 1085-1096.

46 T. Lazaridis, J. Phys. Chem. B, 1998, 102, 3531-3541.

47 Y. Sugita, A. Kitao and Y. Okamoto, J. Chem. Phys., 2000, 113, 6042-6051.

48 M. R. Shirts and J. D. Chodera, J. Chem. Phys., 2008, 129, 124105.

49 H.-J. Woo and B. Roux, Proc. Natl. Acad. Sci. U.S.A., 2005, 102, 6825-6830.

50 J. C. Gumbart, B. Roux and C. Chipot, J. Chem. Theory Comput., 2012, 9, 794-802.

51 J. C. Gumbart, B. Roux and C. Chipot, J. Chem. Theory Comput., 2013, 9, 3789-3798.

52 Z. Shamsi, A. S. Moffett and D. Shukla, Sci. Rep., 2017, 7, 12700.

53 Z. Shamsi, K. J. Cheng and D. Shukla, J. Phys. Chem. B, 2018, 122, 8386-8395.

54 C. Zhao and D. Shukla, Sci. Rep., 2018, 8, 17748.

55 G. M. West, B. D. Pascal, L.-M. Ng, F.-F. Soon, K. Melcher, H. E. Xu, M. J. Chalmers and P. R. Griffin, Structure, 2013, 21, 229-235.

56 W. E and E. Vanden-Eijnden, J. Stat. Phys., 2006, 123, 503.

57 W. E and E. Vanden-Eijnden, Annu. Rev. Phys. Chem., 2010, 61, 391-420.

58 P. Metzner, F. Noé and C. Schütte, Phys. Rev. E, 2009, 80, 021106.

59 K. Haider, L. Wickstrom, S. Ramsey, M. K. Gilson and T. Kurtzman, J. Phys. Chem. B, 2016, 120, 8743-8756.

60 Q. Hao, P. Yin, C. Yan, X. Yuan, W. Li, Z. Zhang, L. Liu, J. Wang and N. Yan, J. Biol. Chem, 2010, 285, 28946-28952.

61 X. Yuan, P. Yin, Q. Hao, C. Yan, J. Wang and N. Yan, J. Biol. Chem, 2010, 285, 28953-28958.

62 T. E. Balius, M. Fischer, R. M. Stein, T. B. Adler, C. N. Nguyen, A. Cruz, M. K. Gilson, T. Kurtzman and B. K. Shoichet, Proc. Natl. Acad. Sci. U.S.A., 2017, 114, E6839-E6846.

63 Y. He, Q. Hao, W. Li, C. Yan, N. Yan and P. Yin, PLoS ONE, 2014, 9, e95246.

64 G. Bai, D.-H. Yang, Y. Zhao, S. Ha, F. Yang, J. Ma, X.-S. Gao, Z.-M. Wang and J.-K. Zhu, Plant Mol. Biol., 2013, 83, 651664.

65 Y.-G. Wang, F.-L. Fu, H.-Q. Yu, T. Hu, Y.-Y. Zhang, Y. Tao, J.-K. Zhu, Y. Zhao and W.-C. Li, Plant Mol. Biol., 2018, 96, 245263. 